\title{
DICKSON CURVES
}

\author{
JAVIER GOMEZ-CALDERON
}

Received 6 March 2006; Accepted 26 March 2006

Let $K_{q}$ denote the finite field of order $q$ and odd characteristic $p$. For $a \in K_{q}$, let $g_{d}(x, a)$ denote the Dickson polynomial of degree $d$ defined by $g_{d}(x, a)=\sum_{i=0}^{[d / 2]} d /$ $(d-i)\left(\begin{array}{c}d-i \\ i\end{array}\right)(-a)^{i} x^{d-2 i}$. Let $f(x)$ denote a monic polynomial with coefficients in $K_{q}$. Assume that $f^{2}(x)-4$ is not a perfect square and $\operatorname{gcd}(p, d)=1$. Also assume that $f(x)$ and $g_{2}(f(x), 1)$ are not of the form $g_{d}(h(x), c)$. In this note, we show that the polynomial $g_{d}(y, 1)-f(x) \in K_{q}[x, y]$ is absolutely irreducible.

Copyright @ 2006 Hindawi Publishing Corporation. All rights reserved.

Let $K_{q}$ denote the finite field of order $q$ and odd characteristic $p$. For $a \in K_{q}$, let $g_{d}(x, a)$ denote the Dickson polynomial of degree $d$ and parameter $a$ defined by $g_{d}(x, a)=\sum_{i=0}^{[d / 2]} d /$ $(d-i)\left(\begin{array}{c}d-i \\ i\end{array}\right)(-a)^{i} x^{d-2 i}$. Alternatively, $g_{d}(x, a)$ can also be defined by the second-order linear recursive sequence

$$
g_{d}(x, a)=x g_{d-1}(x, a)-a g_{d-2}(x, a)
$$

where $g_{o}(x, a)=2$ and $g_{1}(x, a)=x$. Thus,

$$
g_{d}(x, a)=g_{d}\left(y+\frac{a}{y}, a\right)=y^{d}+\frac{a^{d}}{y^{d}},
$$

where $x$ and $y$ are related by the generating equation $y^{2}-x y+a=0$. Dickson polynomials have been extensively studied by many authors and an excellent survey of their many properties and applications has been written by Lidl et al. [2]. Since $g_{d}(x, 0)=x^{d}$, the Dickson polynomial $g_{d}(x, a)$ may be viewed as a generalization of the power polynomial $x^{d}$. Equations of the form $y^{d}=f(x)$ are called elliptic equations and have a very rich research history, see, for example, [1, Chapter 18]. In particular, if $f(x)=\left(x-c_{1}\right)^{d_{1}} \cdots(x-$ $\left.c_{s}\right)^{d_{s}}$ is the factorization of $f(x) \in K_{q}[x]$ in $\bar{K}_{q}$, then it is easy to prove that, see [3, page 11], $y^{d}-f(x) \in K_{q}[x, y]$ is absolutely irreducible if and only if $\operatorname{gcd}\left(d, d_{1}, \ldots, d_{s}\right)=1$. Hence, applying Weil's Riemman hypothesis theorem [3, page 131], if $e=\max \{d, \operatorname{deg}(f(x))\}$ 
and $\operatorname{gcd}\left(d, d_{1}, \ldots, d_{s}\right)=1$, then the number of roots $N$ of $y^{d}-f(x)$ in $K_{q} \times K_{q}$ satisfies the inequality

$$
|N-q| \leq(e-1)(e-2) \sqrt{q}+c(e)
$$

for some constant $c(e)$.

In this note, we show that if $\operatorname{gcd}(p, d)=1$ and $f^{2}(x)-4$ is not a perfect square, then $g_{d}(y, 1)-f(x) \in K_{q}[x, y]$ is absolutely irreducible as far as both $f(x)$ and $g_{2}(f(x), 1)$ are not of the form $g_{d}(h(x), c)$ for some constant $c$ and $h(x) \in K_{q}[x]$.

The following lemmas will be needed to prove our main result, Theorem 3 .

Lemma 1. Let $K_{q}$ denote the finite field of order $q$ and odd characteristic p. Let $f(x)$ be a monic polynomial with coefficients in $K_{q}$. Assume that $f^{2}(x)-4$ is not a perfect square. Then

(a) $\left(f(x) \pm \sqrt{f^{2}(x)-4}\right)^{n} \notin K_{q}(x)$ for $n \geq 1$;

(b) if $n \geq 1$ and $\left(f(x)+\sqrt{f^{2}(x)-4}\right)^{n}+c\left(f(x)-\sqrt{f^{2}(x)-4}\right)^{n} \in \bar{K}_{q}(x)$, then $c=1$.

Proof. (a) Assume that $\left(f(x) \pm \sqrt{f^{2}(x)-4}\right)^{n}=\sum_{j=0}^{n}(-1)^{j}\left(\begin{array}{c}n \\ j\end{array}\right) f^{n-j}(x)\left(\sqrt{f^{2}(x)-4}\right)^{j} \in$ $K_{q}(x)$. Then

$$
h(x)=\sum_{i=0}^{m}\left(\begin{array}{c}
n \\
2 i+1
\end{array}\right) f^{n-2 i-1}(x)(f(x)-4)^{i}=0,
$$

where $m=[(n-1) / 2]$. Hence, the leading coefficient of $h(x)$ gives the contradiction $\sum_{i=0}^{m}\left(\begin{array}{c}n \\ 2 i+1\end{array}\right)=2^{n-1}=0$. Therefore, $\left(f(x) \pm \sqrt{f^{2}(x)-4}\right)^{n} \notin K_{q}(x)$ for $n \geq 1$.

(b) Assume that $\left(f(x)+\sqrt{f^{2}(x)-4}\right)^{n}+c\left(f(x)-\sqrt{f^{2}(x)-4}\right)^{n} \in \bar{K}_{q}(x)$ for some $n \geq$ 1. Then

$$
\sum_{i=0}^{m}(1-c)(f(x))^{n-2 i-1}\left(f^{2}(x)-4\right)^{i}=0,
$$

where $m=[(n-1) / 2]$. Therefore, $(1-c) \sum_{i=0}^{m}\left(\begin{array}{c}n \\ 2 i+1\end{array}\right)=(1-c) 2^{n-1}=0$ and so $c=1$.

Lemma 2. With notation as in Theorem 3, assume that $\sigma_{r}\left(a_{1}, \ldots, a_{n}\right) \theta^{r}+\sigma_{r}\left(1 / a_{1}, \ldots, 1 /\right.$ $\left.a_{n}\right) \theta^{-r} \in \bar{K}_{q}(x)$ for some $r \geq 1$. Then, $\sigma_{r}\left(a_{1}, \ldots, a_{n}\right)=0$ if and only if $\sigma_{r}\left(1 / a_{1}, \ldots, 1 / a_{n}\right)=0$.

Proof. Assume that $\sigma_{r}\left(a_{1}, \ldots, a_{n}\right) \neq 0$ and $\sigma_{r}\left(1 / a_{1}, \ldots, 1 / a_{n}\right)=0$. Then, $\theta^{d r}=(f(x)+$ $\left.\sqrt{f^{2}(x)-4}\right)^{r} \in \bar{K}_{q}(x)$ contradicting Lemma 2. A similar argument also shows that the cases $\sigma_{r}\left(a_{1}, \ldots, a_{n}\right)=0$ and $\sigma_{r}\left(1 / a_{1}, \ldots, 1 / a_{n}\right) \neq 0$ cannot occur. Therefore, $\sigma_{r}\left(a_{1}, \ldots, a_{n}\right)=$ 0 if and only if $\sigma_{r}\left(1 / a_{1}, \ldots, 1 / a_{n}\right)=0$.

Theorem 3. Let $K_{q}$ denote the finite field of order $q$ and odd characteristic p. Let $f(x)$ be a monic polynomial with coefficients in $K_{q}$. Assume that $f^{2}(x)-4$ is not a perfect square. For $d \geq 1$, let $g_{d}(y, 1)$ denote the Dickson polynomial of degree $d$ and parameter 1 . Assume that $f(x)$ and $g_{2}(f(x), 1)$ are not of the form $g_{d}(h(x), c)$ for some $c \in \bar{K}_{q}$ and $h(x) \in K_{q}[x]$. Assume that $\operatorname{gcd}(p, d)=1$. Then, $g_{d}(y, 1)-f(x) \in K_{q}[x, y]$ is absolutely irreducible. 
Proof. Consider $g_{d}(y, 1)-f(x)$ as a polynomial in $y$ with coefficients in the field of rational functions $\bar{K}_{q}(x)$. Set $y=w+1 / w$. Then, $g_{d}(y, 1)-f(x)=w^{d}+1 / w^{d}-f(x)=0$ if and only if $w^{d}=\left(f(x) \pm \sqrt{f^{2}(x)-4}\right) / 2$. Hence, combining with Lemma 1 ,

$$
g_{d}(y, 1)-f(x)=\prod_{i=1}^{d}\left(y-\varsigma_{d}^{i} \theta-\frac{1}{\varsigma_{d}^{i} \theta}\right),
$$

where $\theta$ is any of the roots of $w^{d}=\left(f(x) \pm \sqrt{f^{2}(x)-4}\right) / 2$.

Now assume that $g_{d}(y, 1)-f(x)$ is reducible over $\bar{K}_{q}[x, y]$; that is,

$$
g_{d}(y, 1)-f(x)=\prod_{i=1}^{r} f_{i}(x, y)
$$

for some polynomials $f_{i}(x, y) \in \bar{K}_{q}[x, y]$ with degree in $y$ less than $d$. Then,

$$
f_{i}(x, y)=\prod_{j=1}^{n_{i}}\left(y-a_{i j} \theta-\frac{1}{a_{i j} \theta}\right) \in \bar{K}_{q}[x, y],
$$

where $\left\{a_{i 1}, a_{i 2}, \ldots, a_{i n_{i}}\right\} \subset\left\{1, \varsigma_{d}, \ldots, \varsigma_{d}^{n-1}\right\}$.

Therefore,

$$
\begin{aligned}
f_{i}(x, y) & =\prod_{j=1}^{n_{i}}\left(y-a_{i j} \theta-\frac{1}{a_{i j} \theta}\right) \\
& =y^{n_{i}}+h_{i 1}(x) y^{n_{i}-1}+\cdots+h_{i n_{i}-1}(x) y+h_{i n_{i}}(x) \in \bar{K}_{q}[x, y],
\end{aligned}
$$

where the polynomials $h_{i j}(x)$ can be expressed in terms of elementary symmetric polynomials as the following equations show:

$$
\begin{gathered}
h_{i 1}(x)=\sigma_{1}\left(a_{i 1}, a_{i 2}, \ldots, a_{i n_{i}}\right) \theta+\sigma_{1}\left(\frac{1}{a_{i 1}}, \frac{1}{a_{i 2}}, \ldots, \frac{1}{a_{i n_{i}}}\right) \theta^{-1}, \\
h_{i 2}(x)=\sigma_{2}\left(a_{i 1}, \ldots, a_{i n_{i}}\right) \theta^{2}+\sigma_{2}\left(\frac{1}{a_{i 1}}, \ldots, \frac{1}{a_{i n_{i}}}\right) \theta^{-2} \\
+\sum_{j=1}^{n_{i}}\left[\frac{\sigma_{1}\left(a_{i 1}, \ldots, \hat{a}_{i j}, \ldots, a_{i n_{i}}\right)}{a_{i j}}+a_{i j} \sigma_{1}\left(\frac{1}{a_{i 1}}, \ldots, \frac{\hat{1}}{a_{i j}}, \ldots, \frac{1}{a_{i n_{i}}}\right)\right],
\end{gathered}
$$




$$
\begin{aligned}
h_{i n_{i}}(x)= & \sigma_{n_{i}}\left(a_{i 1}, \ldots, a_{i n_{i}}\right) \theta^{n_{i}}+\sigma_{n_{i}}\left(\frac{1}{a_{i 1}}, \ldots, \frac{1}{a_{i n_{i}}}\right) \theta^{-n_{i}} \\
+ & \sum_{j=1}^{n_{i}}\left[\frac{\sigma_{n_{i}-1}\left(a_{i 1}, \ldots, \hat{a}_{i j}, \ldots, a_{i n_{i}}\right) \theta^{n_{i}-2}}{a_{i j}}+a_{i j} \sigma_{n_{i}-1}\left(\frac{1}{a_{i 1}}, \ldots, \frac{\hat{1}}{a_{i j}}, \ldots, \frac{1}{a_{i n_{i}}}\right) \theta^{-n_{i}+2}\right] \\
+ & \sum_{t \neq j}^{n_{i}}\left[\frac{\sigma_{n_{i}-2}\left(a_{i 1}, \ldots, \hat{a}_{i t}, \ldots, \hat{a}_{i j}, \ldots, a_{i n_{i}}\right) \theta^{n_{i}-4}}{a_{i t} a_{i j}}\right. \\
& \left.+a_{i t} a_{i j} \sigma_{n_{i}-2}\left(\frac{1}{a_{i 1}}, \ldots, \frac{\hat{1}}{a_{i t}}, \ldots, \frac{\hat{1}}{a_{i j}}, \ldots, \frac{1}{a_{i n_{i}}}\right) \theta^{-n_{i}+4}\right] \\
+ & \sum_{t_{s} \neq t_{j}}\left[\frac{\sigma_{n_{i}-w}\left(a_{i 1}, \ldots, \hat{a}_{i t_{1}}, \ldots, \hat{a}_{i t_{w}}, \ldots, a_{i n_{i}}\right) \theta^{n_{i}-2 w}}{a_{i t_{1}} a_{i t_{2}} \cdots a_{i t_{w}}}\right. \\
& \left.+a_{i t_{1}} \cdots a_{i t_{w}} \sigma_{n_{i}-w}\left(\frac{1}{a_{i 1}}, \ldots, \frac{\hat{1}}{a_{i t_{1}}}, \ldots, \frac{\hat{1}}{a_{i t_{w}}}, \ldots, \frac{1}{a_{i n_{i}}}\right) \theta^{-n_{i}+2 w}\right],
\end{aligned}
$$

where $w=\left[n_{i} / 2\right]$ and $\operatorname{deg}\left(h_{i j}(x)\right)<\operatorname{deg}(f(x))$ for $1 \leq j \leq n_{i}$.

Now, combining with Lemma 2, we consider the following two cases.

Case 1. $\sigma_{1}\left(a_{i 1}, \ldots, a_{i n_{i}}\right) \sigma_{1}\left(1 / a_{i 1}, \ldots, 1 / a_{i n_{i}}\right) \neq 0$ for some $1 \leq i \leq r$. Then,

$$
\theta+c_{i} \theta^{-1}=\frac{h_{i 1}(x)}{c_{i}}=H_{i 1}(x) \in \bar{K}_{q}[x],
$$

where $c_{i}=\sigma_{1}\left(1 / a_{i 1}, \ldots, 1 / a_{i n_{i}}\right) / \sigma_{1}\left(a_{i 1}, \ldots, a_{i n_{i}}\right)$. Hence,

$$
\begin{aligned}
g_{d}\left(\theta+\frac{c_{i}}{\theta}, c_{i}\right) & =\frac{f(x) \pm \sqrt{f^{2}(x)-4}}{2}+c_{i}^{d} \frac{f(x) \mp \sqrt{f^{2}(x)-4}}{2} \\
& =g_{d}\left(H_{i 1}(x), c_{i}\right) .
\end{aligned}
$$

Therefore,

$$
f(x)=g_{d}\left(H_{i 1}(x), c_{i}\right)
$$

where $c_{i}^{d}=1$. 
Case 2. $\sigma_{1}\left(a_{i 1}, \ldots, a_{i n_{i}}\right)=\sigma_{1}\left(1 / a_{i 1}, \ldots, 1 / a_{i n_{i}}\right)=0$ for all $1 \leq i \leq r$. Then,

$$
\begin{aligned}
h_{i 2}(x)= & \sigma_{2}\left(a_{i 1}, \ldots, a_{i n_{i}}\right) \theta^{2}+\sigma_{2}\left(\frac{1}{a_{i 1}}, \ldots, \frac{1}{a_{i n_{i}}}\right) \theta^{-2} \\
& +\sum_{j=1}^{n_{i}}\left[\frac{\sigma_{1}\left(a_{i 1}, \ldots, \hat{a}_{i j}, \ldots, a_{i n_{i}}\right)}{a_{i j}}+a_{i j} \sigma_{1}\left(\frac{1}{a_{i 1}}, \ldots, \frac{\hat{1}}{a_{i j}}, \ldots, \frac{1}{a_{i n_{i}}}\right)\right] \\
= & \sigma_{2}\left(a_{i 1}, \ldots, a_{i n_{i}}\right) \theta^{2}+\sigma_{2}\left(\frac{1}{a_{i 1}}, \ldots, \frac{1}{a_{i n_{i}}}\right) \theta^{-2}+\sum_{j=1}^{n_{i}}\left[\frac{-a_{i j}}{a_{i j}}+\frac{a_{i j}}{-a_{i j}}\right] \\
= & \sigma_{2}\left(a_{i 1}, \ldots, a_{i n_{i}}\right) \theta^{2}+\sigma_{2}\left(\frac{1}{a_{i 1}}, \ldots, \frac{1}{a_{i n_{i}}}\right) \theta^{-2}-2 n_{i} .
\end{aligned}
$$

Hence, if $\sigma_{2}\left(a_{i 1}, \ldots, a_{i n_{i}}\right)=\sigma_{2}\left(1 / a_{i 1}, \ldots, 1 / a_{i n_{i}}\right)=0$ for all $1 \leq i \leq r$, then the second-order leading coefficient of $g_{d}(y, 1)-f(x)=\prod_{i=1}^{r} f_{i}(x, y)$ at $y$ gives the contradiction

$$
d=\sum_{i=1}^{r} 2 n_{i}=2 \sum_{i=1}^{r} n_{i}=2 d .
$$

So, $\sigma_{2}\left(a_{i 1}, \ldots, a_{i n_{i}}\right) \sigma_{2}\left(1 / a_{i 1}, \ldots, 1 / a_{i n_{i}}\right) \neq 0$ for some value $i$ and consequently, using such particular value,

$$
\theta^{2}+c_{i} \theta^{-2}=\frac{h_{i 2}(x)+2 n_{i}}{c_{i}}=H_{i 2}(x) \in \bar{K}_{q}[x]
$$

where $c_{i}=\sigma_{2}\left(1 / a_{i 1}, \ldots, 1 / a_{i n_{i}}\right) / \sigma_{2}\left(a_{i 1}, \ldots, a_{i n_{i}}\right)$. Therefore,

$$
\begin{gathered}
g_{d}\left(\theta^{2}+\frac{c_{i}}{\theta^{2}}, c_{i}\right)=\left(\frac{f(x) \pm \sqrt{f^{2}(x)-4}}{2}\right)^{2}+c_{i}^{d}\left(\frac{f(x) \mp \sqrt{f^{2}(x)-4}}{2}\right)^{2}=g_{d}\left(H_{i 2}(x), c_{i}\right), \\
g_{2}(f(x), 1)=g_{d}\left(H_{i 1}(x), c_{i}\right),
\end{gathered}
$$

where $c_{i}^{d}=1$.

Since both cases contradict our assumptions on $f(x)$ and $g_{2}(f(x), 1)$, then we conclude that $g_{d}(y, 1)-f(x)$ is absolutely irreducible.

Corollary 4. With conditions as in Theorem 3, let $N$ denote the number of zeros of $g_{d}(y, 1)$ $-f(x)$ in $K_{q} \times K_{q}$. Let $e=\max \{d, \operatorname{deg}(f(x))\}$. Then,

$$
|N-q| \leq(e-1)(e-2) \sqrt{q}+c(e)
$$

for some constant $c(e)$.

Proof. Combine Theorem 3 and Weil's Riemann hypothesis theorem for curves over finite fields. 


\section{Dickson curves}

\section{References}

[1] K. F. Ireland and M. I. Rosen, A Classical Introduction to Modern Number Theory, Springer, New York, 1998.

[2] R. Lidl, G. L. Mullen, and G. Turnwald, Dickson Polynomials, Pitman Monographs and Surveys in Pure and Applied Mathematics, vol. 65, Longman Scientific \& Technical, Harlow, 1993.

[3] W. M. Schmidt, Equations Over Finite Fields. An Elementary Approach, Lecture Notes in Mathematics, vol. 536, Springer, Berlin, 1976.

Javier Gomez-Calderon: Department of Mathematics, The Pennsylvania State University,

New Kensington Campus, New Kensington, PA 15068, USA

E-mail address: jxg11@psu.edu 


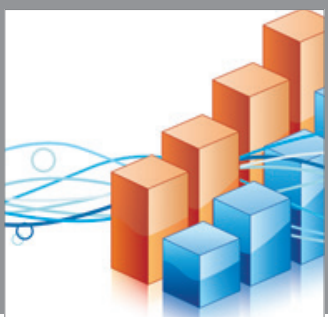

Advances in

Operations Research

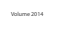

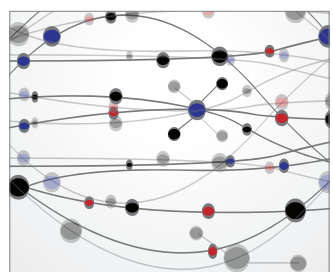

\section{The Scientific} World Journal
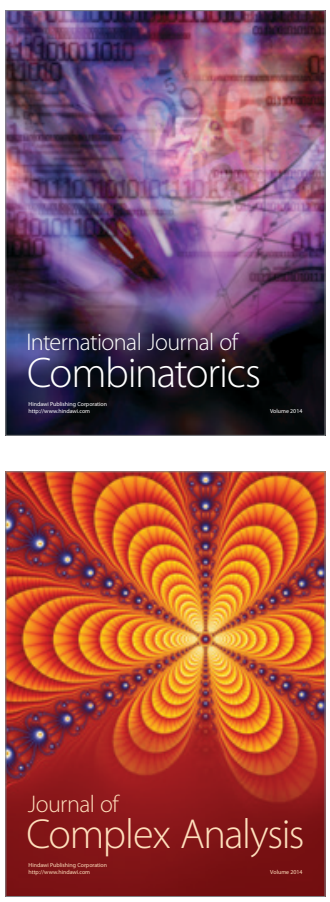

International Journal of

Mathematics and

Mathematical

Sciences
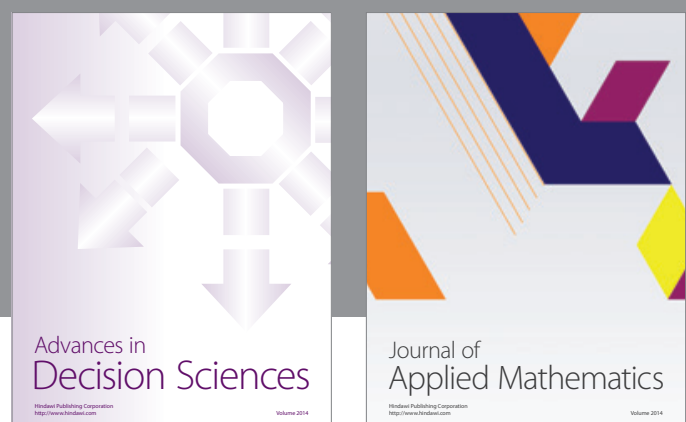

Journal of

Applied Mathematics


Submit your manuscripts at http://www.hindawi.com


Mathematical Problems in Engineering


Journal of

Function Spaces


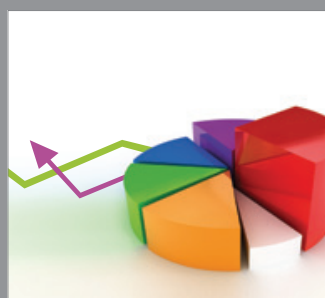

ournal of

Probability and Statistics

Promensencen
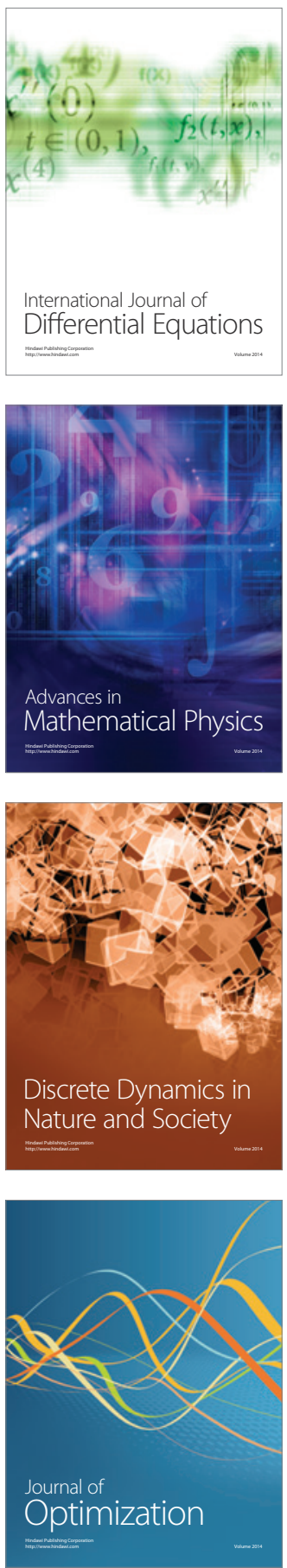